\title{
Design of the Train Data Processing Center in Intelligent Train
}

\author{
Yanmei Guo ${ }^{1+}$, Yifeng Lin $^{2}$, Xiangxian Chen ${ }^{1}$ \\ Department of Instrumentation Science and Engineering, Zhejiang University, Hangzhou, China \\ ${ }^{2}$ Research and Development Center, Zhejiang United Science and Technology Co., Ltd, Hangzhou, China
}

\begin{abstract}
In current train network control system, the key performance parameters of monitoring quantity is few, and the data analysis ability is weak. In transit fault analysis and self-learning are also unable. Most of the train faults can only be reproduced and handed with through the test and line checking when the EMU run back to the library. To address the problem above, we have developed a set of dynamic data sensing system based on Internet of Things technology and sensor network technology, that is, the train data center with data records, in transit warning, train transmission and other functions. The system can be more comprehensive and more systematic to control EMU operating status, to achieve in transit warning and timely guidance, and to take the purpose of emergency measures reasonably.
\end{abstract}

Keywords: the train data center, in transit warning, data storage

\section{Introduction}

The data center of urban rail transit is the basic platform of operation monitoring, emergency command and information management for subway enterprise. It mainly completes the acquisition and sharing of data from various platforms, such as heterogeneous data, professional monitoring systems and operation systems. Information query, interactive and other functions are also included [1].

Intelligent high-speed train system takes the high-speed train as the core, the holographic train state perception and dynamic digital operating environment as the basis, and the information intelligence processing and interaction as the support, with self-test, self-diagnosis and decision-making capacity. The intelligent train obtains the environmental data of the train running when the train is running by operating the environment perception system, and the state data of the train running through the sensor network constructed by the sensor, and the history data of the main equipment of the train through the electronic tag [2].However, the current ground data center has a delay, and it couldn't achieve in transit warning. Fault handing capacity is also weak. Therefore, it is necessary to establish an intelligent train system to meet the current requirement [3].

Based on the original CRH380 series of high-speed train, the intelligent train described in this paper increases the train intelligent system. The new intelligent system includes four main parts, train intelligent sensing network, train data processing center, train wireless communication system and train passenger service system. This paper mainly studies the train data processing center. That is the dynamic data sensing system which is based on Internet of things technology and sensor network technology, and it has the functions of data recording, in transit warning and the "train-to-ground" transmission.

\section{The Train Data Processing Center}

At present, the train network control system is mainly used for the control of train operation, the monitoring of the condition of the train equipment, the diagnosis and data storage of the train. The

\footnotetext{
Corresponding author. Tel.: + 8617367078393.
}

E-mail address: gym12840@163.com. 
monitoring of the key performance parameters are few and the data analysis ability is weak. In transit fault analysis and self-learning cannot be realized. Most of the train faults can only be reproduced and handed with through the test and line checking when the EMU run back to the library. Through the establishment of intelligent systems, intelligent train stores the historical fault database, EMU important performance parameters and emergency measures in the train data center. With the help of the fault diagnosis expert system designed in the train data center, the fault location will be more accurate, and the fault diagnosis information will be richer, and finally the train make in-train diagnosis and in transit warning come true. At the same time, the train data center display can show the faults of the equipment, time of faults, line location, related parameters and other information, including the data recorded and sound and light alarm. Also, the train center can provide operational guidelines for conductors through text, pictures, images and other forms, which can improve the capacity of emergency management personnel.

The train data center developed by this project can be used for "the train intelligent monitoring system in high-speed train". The use of high-performance stacking computing architecture, and fault-based tree expert diagnostic system, and large capacity storage capacity are the basic technical characteristics of the system. The working principle of this system can be summarized as follows: through the train bus, Ethernet and other networks, it has a real-time collection and storage of train sensor information and train equipment status information, and makes judgments by the health status of components of the train. If the judge fails, we do a rapid fault derivation firstly with the help of expert knowledge base, find the source of the fault, and propose a solution. When faced with the situation of some fault which can not be detected by sensors and equipment, the system will quickly diagnose fault by the conductor by hand to manually select the input method. All the fault derivation process and the results can be provided in a friendly man-machine interface to facilitate the operation of the conductor.

The train data processing center has five functional modules [4]:

(1) Data access: data access and integration of column control network, sensor network, tag information;

(2) Data storage: to achieve the classification and storage of train dynamic and static data ;

(3) Data processing: to generate in transit warning and alarm information;

(4) Data show: providing application interface for the conductor;

(5) Data transmission: monitoring data of the "train -to- ground" transmission.

\section{Overall Architecture Design of System}

The train data processing center mainly consists of two parts, the train data center host and display terminal. The train data processing center host is the electronic equipment which helps intelligent train with data centralized processing, in transit warning, unified transmission. The main functions of the train data processing center display are the basic information display, the running data display, the in transit warning and alarm, the historical fault inquiry, the emergency fault handling guide, the artificial fault entry and the component resume information.

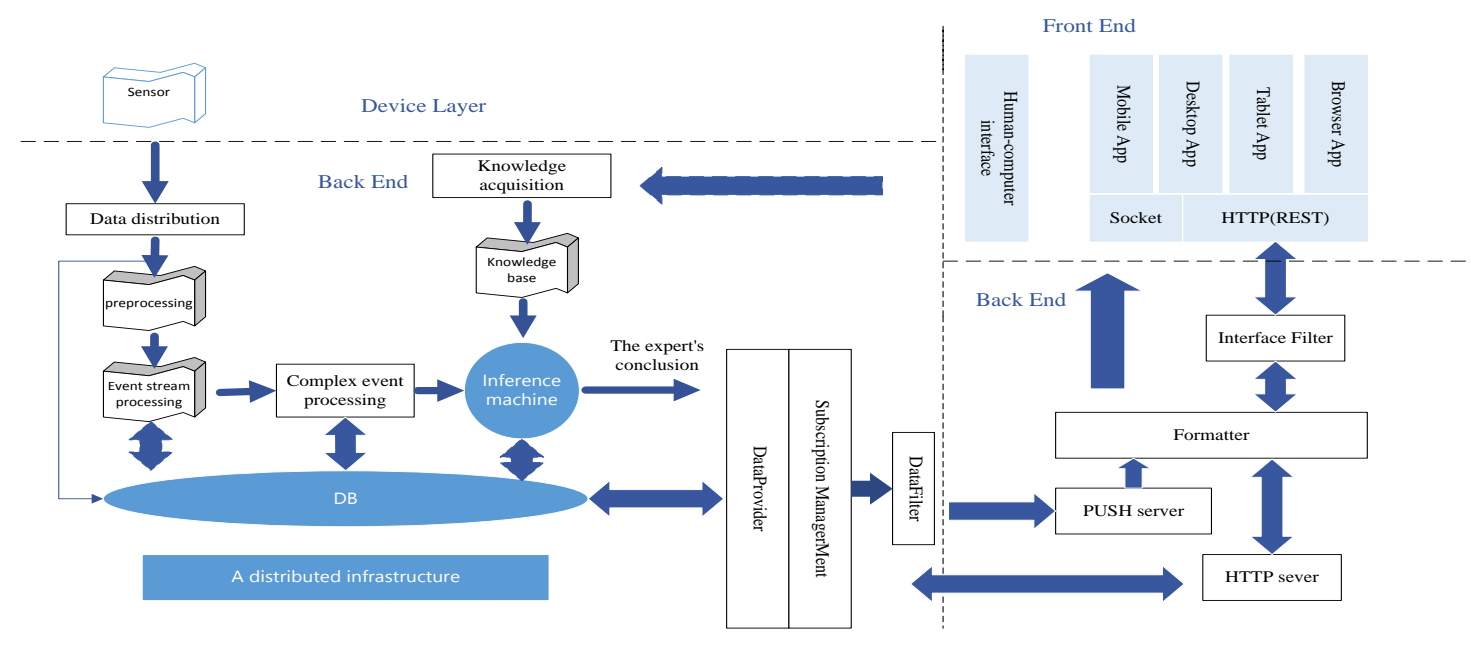


The project uses industrial-grade high-performance bus technology and high-performance computing technology to build a high-performance, large storage capacity of the hardware platform. In addition, combined with the train intelligent diagnostic expert system, the system can also determine the cause of the fault with the use of expert knowledge base. In addition to the expert system, the system also completes the access and storage of the sensing information, and involves the development of the user interface to fully meet the needs of human-computer interaction. The train data center architecture design is shown in Figure 1.

\section{Hardware Design of the Train Data Center}

The hardware structure of the train data center developed by this project, which is applicable to high speed / subway train intelligent diagnosis system, is the basic hardware architecture of 3U CPCI. In addition to the CPCI bus, the backboard has an Ethernet bus for the collection, storage, intelligent analysis and decision making of high-speed train.

CPCI bus has the advantage of low cost, strong seismic performance. However, CPCI bus is a sharing-type bus, which means that if the bus type is $32-b i t * 33 \mathrm{Mhz}$, the total bandwidth of the slot shared by the CPCI bus is only about $1 \mathrm{G}$ bps.

$\mathrm{GbE}$ Ethernet bus has the feature of point to point transmission, strong transmission capacity, and the interface is relatively cheap. The system uses $8 \mathrm{GbE}$ switchboard as the system's Ethernet access and internal data exchange. The introduction of the Ethernet bus is mainly used to provide large bandwidth access and improve the system's internal communication bandwidth. For large amounts of accessing data, such as video data, the convergent sensor data from the front, can be transmitted and processed over Ethernet.

The system supports the board types such as the main control calculation board, the storage board, and the exchange board [5].

(1) The main control calculation board: the stack of parallel computing to complete the access of business management and analysis.

(2) The storage board: the storage of the business data, which is mainly sensor data at present. It is mainly divided into two types, the hard disk carrier board and a storage board.

(3) Exchange board: exchange and storage of internal data.

\section{Software Design of Train Data Center}

The software consists of three modules: data acquisition module, data processing module, network sending module [6], the overall structure of the system and data flow are shown in Figure 2.

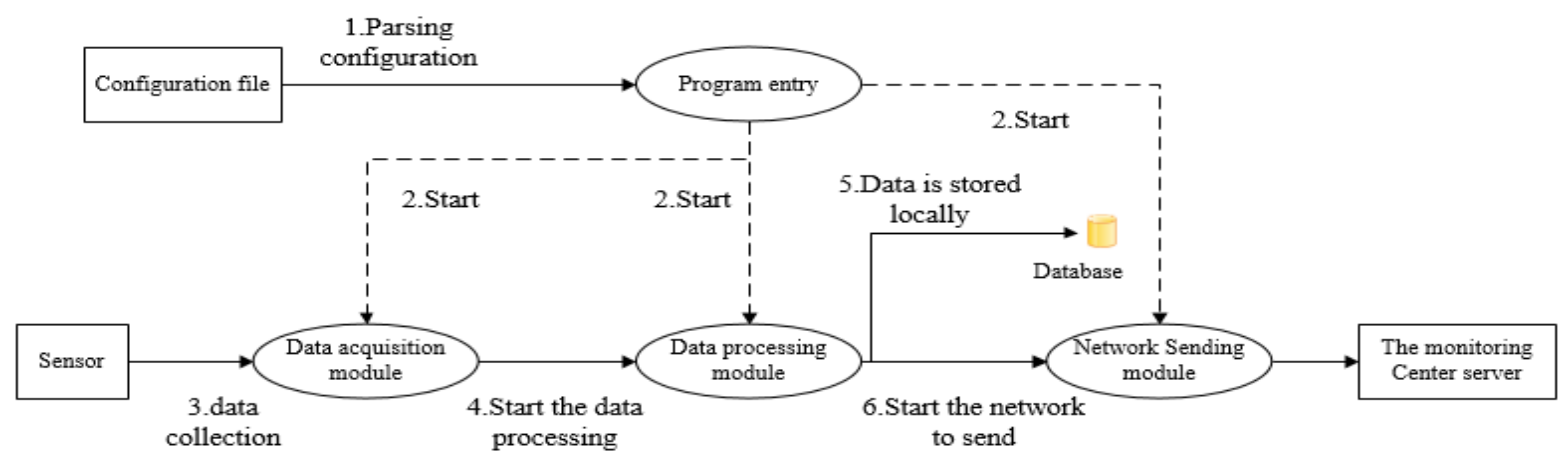

Fig. 2: Overall structure of Software in the train data center

The data acquisition module can realize the data communication with the sensor as well as the protocol analysis to obtain the required physical quantity. Data collection divides into various types depending on the type of sensor data, which includes periodic acquisition tasks (such as axle box temperature, axial pressure, etc.), as well as triggering acquisition tasks (such as infrared intrusion).The data acquisition module is 
implemented by the event-driven model, so that the acquisition task can get the quick response and reduce the occupation of the system resources. The concrete flow is shown in Figure 3.

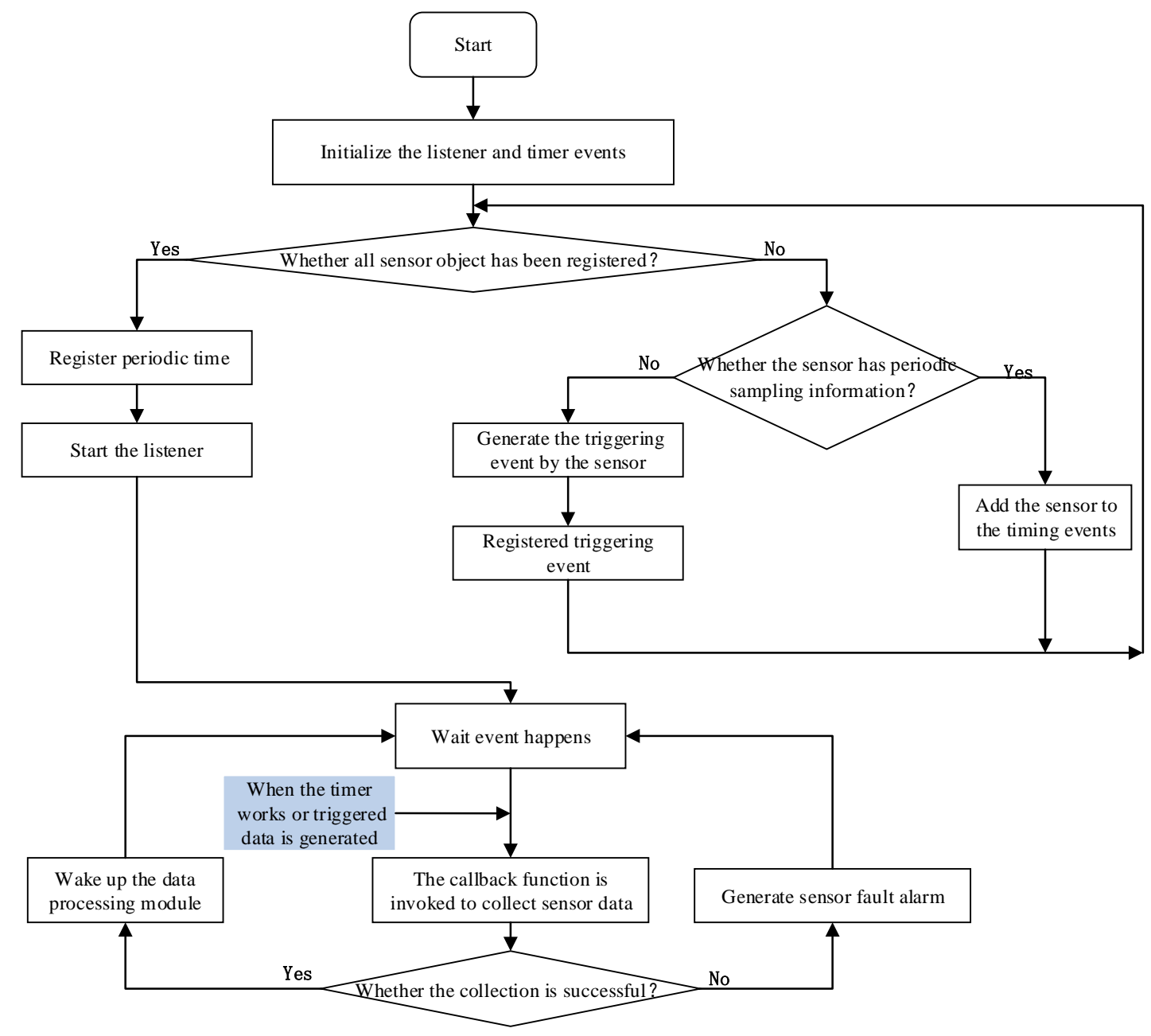

Fig. 3: Data acquisition flow chart

The data processing module is responsible for a series of algorithmic processing and data analysis of the acquired raw data, resulting in the state data which is needed to upload in the final. If the status data is abnormal, the corresponding alarm data is generated, and the control signals output to different external objects according to the different alarm levels. At the same time, to meet the needs of data tracing, and to prevent data loss caused by the accidental collapse of the system, the system needs to use the database to form a persistence storage for the data within a certain length.

The network sending module carries on the serialization processing and the encryption processing to the state data and the alarm data, and finally realizes the network data communication between the central monitoring server and the train control system according to the internal application layer communication protocol and the TCP protocol.

\section{Summary}

In this paper, taking the train data center as the object, we have studied the system architecture, hardware, software features. The train data center designed by this project realizes the functions of basic information and running data display, in transit warning and alarm, historical fault query, emergency fault handling guide, manual fault entry and spare parts resume information. It provides a data source and guarantee of communication for train ground monitoring, which has a certain reference function for the comprehensive promotion of intelligent train safety monitoring system.

\section{Acknowledgements}

This work is supported by the funding from the National Science and Technology Infrastructure Program 
of China under Grant 2015BAG19B03.

\section{References}

[1] Liu Wei, Zhang Ming, Liu Yangxue, Application of Data Center for Urban Rail Transit Based on Cloud Platform [J] computer applications, 2016,25 (9), 72-78.

[2] Li Xiaojun. Design and research of Intelligent high-speed train program[A]. China Intelligent Transportation Association. The Eighth Annual Meeting Proceedings of China Intelligent Transportation - Rail Traffic [C]. China Intelligent Transportation Association: 2013.

[3] DING Rong-jun, YANG Wei-feng, TANG Jun. Study and Application of Intelligent Technology of Rail Transit Equipment [J]. Electric Drive, 2012, (05).

[4] Shen Chongcheng, Li Peng, Wu Donghua, etc. Overall design of train intelligent system in High-speed train [J]. Electronic Technology and Software Engineering, 2013 (20): 32-33.

[5] Xu Ping.Design and Implementation of Train Data Center and Ground Receiving Gateway [D]. Electronic Technology of Guilin University, 2015.

[6] Zhang Youbing, Ma Lin, Niu Daoheng, etc. Software Design of Controlled Train Data Center [J]. Journal of Railway Communications, 2015, 51 (4): 56-59. 